\section{Schülerprojekt Katastrophenschutz: Film ab!}

— Ein interessantes Projekt wurde Ende vergangenen Jahres am Schulzentrum für Gesundheitsberufe am Niederrhein $\mathrm{GmbH}$ (SGN) - es bildet für zwei Krankenhäuser von Mönchengladbach in der Gesundheits-, Kranken- und Kinderkrankenpflege aus - gestartet. Mit 45 Auszubildenden wurde ein Film zum ManV (Massenanfall von Verletzten) im Krankenhaus gedreht. Der Anstoß für das Projekt kam von der Arbeitssicherheitsbeauftragten des Krankenhauses. Ziel war es, sich mit der praktischen Umsetzung der Katastrophenpläne in den Kliniken zu befassen. Es entstand die Idee eines Filmes. Die Kurse schrieben unter Begleitung der Lehrer in Eigenarbeit und unter Verwendung der Katastrophenpläne ein Drehbuch. Die Ergebnisse wurden in der gesamten Gruppe abgestimmt, bis ein fertiges Drehbuch vorlag. „Darauf folgten insgesamt drei Drehtage, an denen in unserem Schulzentrum und einem der Krankenhäuser ein Amoklauf nachgestellt wurde und dann die Abläufe, die in einem Krankenhaus bei einer solchen Katastrophensituation passieren, gefilmt wurden", berichtet Sabrina Thomauske, Lehrerin Pflege und Gesundheit am SGN. „Die Schüler haben alles selbstständig organisiert und gemacht - vom Schminken bis zu dem Schneiden der Szenen. Das Resultat ist ein achtminütiger Film, der äußerst gut gelungen ist", so Margit Abt-Kehls von SGN. Neben dem hervorragenden Filmergebnis hätten sich die Kurse besser kennen gelernt. Zudem wurden die theoretischen Inhalte wie von selbst verinnerlicht. Der Film wird bei Mitarbeiterschulungen verwendet.

www.youtube.com/watch?v=kfeMyTIR4gE\&feature=youtu.be

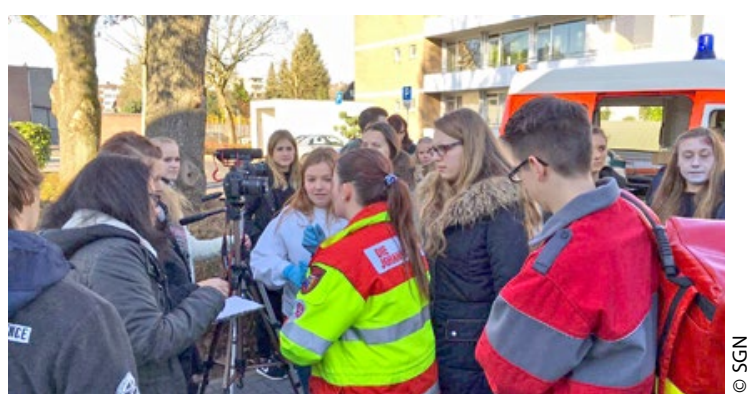

Bundestag

\section{Verbandmittel-Versorgung gesichert}

— Mit der Gesetzesänderung zur Wundversorgung im Heil- und Hilfsmittelversorgungsgesetz sichert der Bundestag auch die Verbandmittel-Versorgung von über 900.000 Menschen mit schweren chronischen Wunden, konstatiert die Initiative für moderne und phasengerechte Wundversorgung im Bundesverband Medizintechnologie (BVMed). Der Gesetzgeber hat eine Definition geschaffen, die die meisten etablierten Verbandmittel einschließt, die heute in der tagtäglichen Patientenversorgung eingesetzt werden, heißt es. Das Fehlen einer Verbandmittel-Definition habe zu wiederkehrenden Konflikten zwischen den Kostenträgern und den Herstellern über die Erstattung spezieller Wundauflagen geführt. Der Gesetzgeber gewährleiste damit auch die Erstattung für Wundverbände zur hydroaktiven Wundbehandlung.

www.info-wundversorgung.de

\section{Kommunikation \\ Interkulturelles Arbeiten}

- An der Charité - Universitätsmedizin Berlin erhielten kürzlich die Absolventen der Fortbildung „Interprofessionelles und Interkulturelles Arbeiten (IPIKA) “ ihre Zertifikate. Ziel der berufsbegleitenden Weiterqualifizierung für Beschäftigte in Medizin, Pflege und Sozialdienst ist es, die interkulturellen Kompetenzen berufsgruppenübergreifend zu stärken. Berlin ist mit rund einer Million Einwohnern mit Migrationshintergrund von einer wachsenden kulturellen, ethnischen und religiösen Vielfalt geprägt. Im Klinikalltag zeigt sich dies durch eine hohe Anzahl von Patienten unterschiedlichster Herkunft sowie internationales Klinikpersonal. „Die Kenntnisse kulturell geprägter Regeln und Normen sowie das Wissen um migrationsbedingte Zugangsbarrieren sind im Gesundheitswesen mittlerweile eine unerlässliche fachliche und soziale Kompetenz", sagt Charité-Projektkoordinatorin Baharan Naghavi. IPIKA schult die Teilnehmer in fünf je zweitägigen Modulen in Patientenkommunikation, Zuwendung und Behandlung.

www.charite.de

Ideen gefragt

\title{
Sepsis CamPetition 2017 startet
}

— Anlässlich des Welt-Sepsis-Tages, der sich am 13. September zum fünften Mal jährt, starten Jenaer Organisationen und Institutionen den Kurzfilmwettbewerb Sepsis CamPetition. Gesucht sind kreative Filmschaffende und Nachwuchstalente, die im Videoformat ideenreich über Sepsis in- formieren wollen. Bis zum 1. Juli können Wettbewerbsbeiträge eingereicht werden, es winken Geld- und Sachpreise. Von Jena ausgehend wird seit 2012 am 13. September jedes Jahr der Welt-Sepsis-Tag begangen. Das Ziel: Sepsis muss bekannter werden. „Während wir anfangs vor allem darauf ge- zielt haben, die allgemeine Aufmerksamkeit auf das Thema Sepsis zu lenken, möchten wir nun Menschen dazu anregen, sich auch stärker inhaltlich mit dieser noch immer weitgehend unbekannten Krankheit auseinanderzusetzen", so Margit Leitner, Koordinatorin des Sepsis CamPetition Wettbewerbs für das Center for Sepsis Control and Care am Universitätsklinikum Jena.

www.uniklinikum-jena.de 